Meta

Journal des traducteurs

Translators' Journal

\title{
Modèles sémantique et propositionnel de l'analyse de la fidélité en traduction
}

\section{Jeanne Dancette}

Volume 37, numéro 3, septembre 1992

URI : https://id.erudit.org/iderudit/002450ar

DOI : https://doi.org/10.7202/002450ar

Aller au sommaire du numéro

Éditeur(s)

Les Presses de l'Université de Montréal

ISSN

0026-0452 (imprimé)

1492-1421 (numérique)

Découvrir la revue

Citer cet article

Dancette, J. (1992). Modèles sémantique et propositionnel de l'analyse de la fidélité en traduction. Meta, 37(3), 440-449. https://doi.org/10.7202/002450ar d'utilisation que vous pouvez consulter en ligne.

https://apropos.erudit.org/fr/usagers/politique-dutilisation/ 


\section{MODÈLES SÉMANTIQUE ET PROPOSITIONNEL DE L'ANALYSE DE LA FIDÉLITÉ EN TRADUCTION}

JEANNE DANCETTE

Université de Montréal, Montréal, Canada

\section{INTRODUCTION}

Pour tenir un langage rigoureux sur la fidélité en traduction, il faudrait disposer de paramètres objectifs de «mesure» du sens qui nous permettent d'établir et de mesurer l'équivalence traductionnelle. Il faudrait donc avoir les moyens, d'une part, de représenter de manière formelle le sens exprimé dans le texte source et, d'autre part, de pondérer la gravité de la faute de sens quand il y a écart dans le texte cible par rapport au sens exprimé dans le texte source, car toutes les unités de sens n'ont pas le même poids dans leur contribution au sens du message que constitue le texte à traduire (TAT).

Au risque d'en décevoir plus d'un, nous dirons, à l'issue d'une étude de données empiriques sur la compréhension en traduction (Dancette 1990), que les notions de mesure du sens et donc de fidélité en traduction sont floues et le resteront probablement encore longtemps, essentiellement du fait que le sens lui-même - avec ses nombreuses composantes complexes - est une donnée difficilement formalisable. Par contre, il est possible d'élaborer quelques outils méthodologiques permettant de mesurer certains des aspects principaux du sens en l'absence desquels on ne peut même pas parler de fidélité. Nous en exposons ci-dessous trois grands types: les réseaux sémantiques, le découpage du texte en unités de sens et la pondération des unités en fonction du contenu macrostructurel de l'information.

La mesure de la fidélité s'articule, en effet, sur au moins trois points, qui feront chacun l'objet d'un développement:

1. Ia formalisation du sens des phrases : nous prendrons comme modèle de représentation les réseaux sémantiques tels que définis par Mel'čuk $(1978,1981)$ dans le modèle sens-texte;

2. le découpage du texte source et du texte cible (de préférence, le même découpage !): on évoquera les problèmes que soulève la segmentation des TAT en unités ;

3. l'appréhension du sens: nous distinguerons le sens global (macrostructurel) du sens du détail (microstructurel); cette distinction nous servira de critère de pondération de la gravité des fautes ${ }^{2}$.

Signalons que les réflexions qui suivent s'inscrivent dans le prolongement de notre article sur la faute de sens en traduction (Dancette 1989), où nous présentons les fondements théoriques de la mesure de la compréhension en traduction. Ce qui suit est plus spécifiquement axé sur quelques aspects circonscrits et pratiques de la question.

\section{RÉSEAUX SÉMANTIOUES}

\section{Formalisation (partielle) du sens}

Cette première partie, où nous présentons les réseaux sémantiques comme outils de représentation du sens, a valeur en quelque sorte de démonstration sémantico-linguistique de la fidélité ou de la non-fidélité en traduction. Nous partons de l'hypothèse (Kintsch et 
van Dijk 1975, 1983) qu'un texte est une suite de propositions articulées véhiculant de l'information; les propositions sont exprimées en termes de prédicats et d'arguments. Le prédicat est l'unité de discours qui indique le procès (exprimé par un verbe, un verbe nominalisé, un adjectif, une préposition ou un connecteur logique, tel que ET, OU, NI). L'argument, aussi appelé actant, est l'unité qui désigne l'être ou la chose qui, d'une manière ou d'une autre, participe au procès.

Dans le modèle de Mel'čuk, les relations prédicatives exprimées dans les propositions sont représentées à l'aide de réseaux sémantiques. Ainsi, la phrase (1)

(1) Pierre a vendu sa voiture à Jacques, pour le prix $x$

aura comme représentation le graphe (1):

$$
\begin{array}{ccc}
\multicolumn{3}{c}{\text { Graphe (1) }} \\
\text { 'Pente' } & \\
\text { 'Pierre' } & \text { 'Jacques' } \quad \text { 'voiture' 'x (prix)' }
\end{array}
$$

Du prédicat vente dépendent quatre arguments dont nous pouvons préciser le rôle casuel: Pierre (agent de la transaction), Jacques (destinataire de la transaction), voiture (objet de la transaction, qu'il faudrait rattacher à Pierre par une autre relation prédicative indiquant la propriété avant la transaction), et $x$ (prix).

Nous dirons, en première approximation, qu'un texte est fidèle par rapport à un texte source si les deux réseaux sont conformes ${ }^{3}$.

Nous voyons ici que la représentation sémantique d'un énoncé suppose une certaine abstraction, puisque le réseau exprime un sens plus ou moins détaché de la forme et que le même réseau peut représenter le sens de nombreuses phrases synonymiques qui véhiculent soit le même contenu propositionnel, en l'occurrence, pour reprendre notre exemple, «La vente par Pierre de sa voiture à Jacques pour le prix de X», soit les mêmes aspects d'une réalité pragmatique: «Jacques a acheté la voiture de Pierre pour le prix de $x »$. Signalons qu'il s'agit là d'un trait particulièrement intéressant pour la traduction; nous y reviendrons plus loin.

Comment le modèle s'applique-t-il pour la représentation du sens d'une phrase plus complexe?

Soit la phrase (2):

(2) This is a year of price competition on color-TV, with the Japanese suffering somewhat from the yen revaluation but fighting hard with high-quality products. (Phrase extraite d'un article de Fortune traitant de la concurrence entre les États-Unis et le Japon, reproduite dans Bénard et Horguelin, 1979.)

Le réseau sémantique fait apparaître les rapports liant le prédicat (competition) à ses arguments (1) et (2) (les participants), (3) (la nature de competition), (4) (l'objet de competition).

\section{Graphe (2)}

$\begin{array}{ccccc} & & & & \text { competition } \\ \text { US } & 1 & 2 & 3 & 4 \\ \text { (non spécifié) } & & \text { Japan } & \text { price } & \text { color-TV }\end{array}$


De plus, on indiquera le temps du procès exprimé par: this is a year:

\section{Graphe (3) \\ time}

2

time of enunciation
1

competition

Le réseau qui suit est une représentation sémantique simplifiée de la phrase (2).

\section{Graphe (4)}

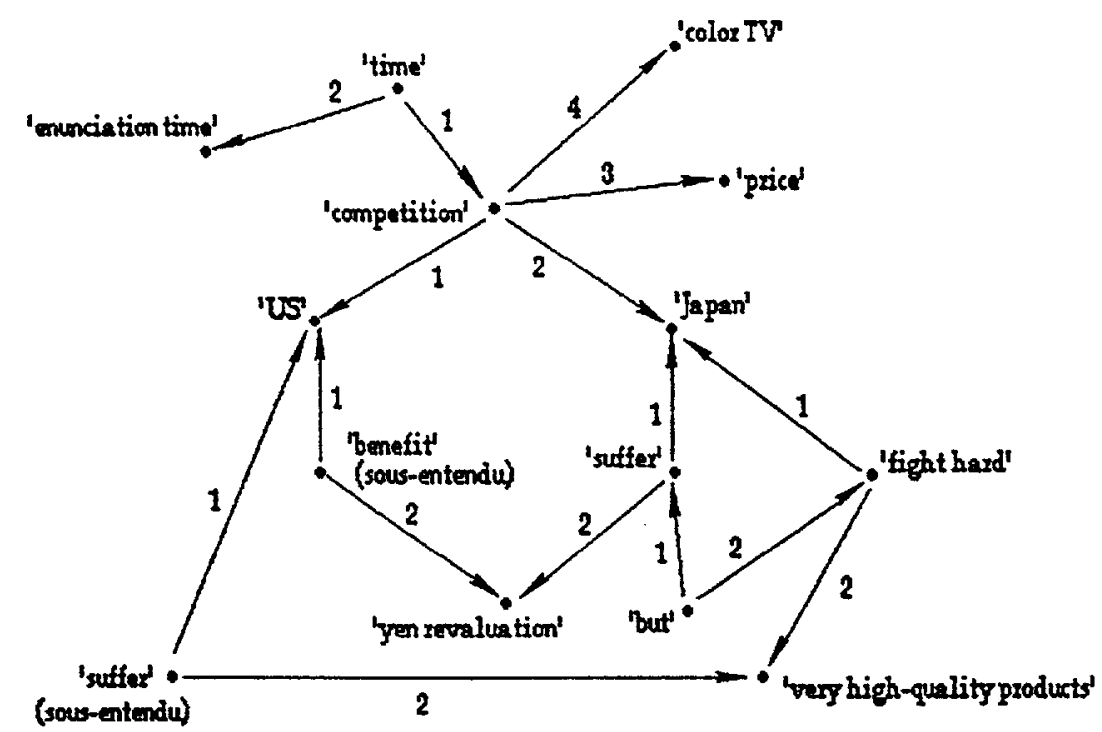

Représentation (simplifiée) de la phrase This is a year of price competition on color-TV, with the Japanese suffering somewhat from the yen revaluation but fighting hard with high-quality products.

Pour que cette représentation sémantique soit plus complète, il faudrait y ajouter la structure rhétorico-communicative (qui permettrait, entre autres, de distinguer le thème (information nouvelle) du rhème (information supposément connue).

\section{Intérêt du réseau sémantique en traductologie}

L'avantage que nous voyons au réseau est son degré d'abstraction. Les étiquettes et les relations prédicatives sont détachćes de leur structure syntaxique, et même, dans une certaine mesure, indépendantes des mots de la phrase. (La préposition with de la phrase (2), par exemple, ne figure pas sur le graphe.)

Nous utilisons les réseaux sémantiques en classe de traduction comme outil pédagogique pour éviter le piège de la traduction servile et encourager le travail sur les concepts plutôt que sur les mots. En effet, des débutants - moins formés à l'exercice d'extraction des concepts (Delisle 1984) - vont généralement produire une traduction du 
genre C'est l'année de la concurrence sur les téléviseurs couleur avec les Japonais qui souffrent de la réévaluation du yen mais qui ripostent avec des produits haut de gamme. Et cela, même quand la consigne est clairement donnée de faire principalement ressortir le sens de la phrase, en se situant au niveau des concepts plutôt que des mots. Si, en revanche, on utilise le réseau comme point de départ d'un travail de réflexion, on peut arriver à des traductions libérées de l'emprise des mots du texte source et riches par leur forme expressive. Les éléments du réseau peuvent, en effet, être «retournés dans tous les sens» et se combiner de mille et une façons ${ }^{4}$.

\section{Limites des réseaux sémantiques comme modèles de représentation du sens}

$\mathrm{Si}$, dans cette partie, nous avons démontré que la fídélité d'un texte traduit peut se mesurer à l'adéquation de son réseau sémantique à celui du TAT, il convient, toutefois, de dégager les limites de ce type d'outil.

- Premièrement, il ne permet pas de rendre facilement compte des effets rhétoriques ou esthétiques du langage, de la forme donc qui est parfois «partie intégrante du message» (Nida et Taber 1971). Par exemple, si nous donnons à l'expression Ah! t'es malin! la représentation sémantique correspondant à $T u$ es un imbécile ou en anglais You're silly, elle ne sera peut-être pas fausse (selon le contexte), mais certainement insuffisante.

Nous sentons le besoin de rajouter des indications sur les figures de style (ironie, sarcasme, jeu de mots), car ces figures contiennent des nuances de sens extrêmement importantes. Une telle sophistication est possible, mais actuellement il n'y a pas de modèle rendant adéquatement et précisément compte des effets stylistiques. Le réseau, tel que nous l'employons, est donc une représentation simplifiée du sens, car il ne rend pas tous les aspects du sens; toutefois, nous estimons qu'il suffit dans de nombreux cas pour rendre compte d'une des composantes essentielle du sens : le contenu propositionnel (au sens de proposition logique) d'un texte.

- La deuxième limite tient au fait que le réseau sémantique ne résout pas le problème méthodologique du sens exact des étiquettes (nœuds) et de leur équivalence sémantique d'une langue à l'autre. Les réseaux doivent donc être utilisés conjointement avec les analyses componentielles du sens des unités sémantiques (Pottier 1963). Mais comment vérifier l'équivalence d'une étiquette si le découpage en unités de sens est différent d'une langue à l'autre? C'est l'objet de notre deuxième partie.

\section{DÉCOUPAGE DU TEXTE}

En ce qui concerne les unités de découpage des textes, il faut, à notre avis, distinguer: 1) la segmentation des textes en tant qu'objets linguistiques porteurs de sens (unités morphologiques, syntaxiques, sémantiques, unités discursives et textuelles);2) le découpage des textes en unités de travail, dans le processus de la traduction. C'est cette deuxième classe que nous appelons UNITÉ DE TRADUCTION, comme synonyme d'unité de traitement, et que nous définissons comme l'unité minimale qui reçoit une équivalence en bloc. Mais comme la traduction (opération) est un processus individuel, probablement non normalisé, dont le succès dépend de critères de performance très personnels (connaissance de tel ou tel idiome, accès plus ou moins rapide aux ressources expressives de la langue cible, etc.), les frontières de l'«unité de traduction» varient d'un individu à l'autre. La notion est donc beaucoup trop subjective pour être retenue pour la mesure de la fidélité.

En revanche, l'UNITÉ DE SENS, définie comme un objet linguistique porteur de sens, est une unité objective pouvant servir de paramètre pour la mesure de la fidélité. Elle peut correspondre à un mot, à une expression ou à un seul trait morphologique 
(1'aspect du verbe, par exemple) ou encore à une phrase entière. Prenons l'expression It is easy to be wise after an event, suivie de but $X$ can rightly say $Y$, qu'on pourrait traduire par: «C'est facile de prédire le passé.» Nous pensons qu'une expression de ce genre, extraite d'un énoncé en situation de discours, se décode de deux façons au moins : par les mots qui la composent; et aussi par la fonction rhétorique qu'elle prend dans le texte, à cause de l'implication «X n'a pas beaucoup de mérite à savoir $Y$ ».

Nous pouvons dire qu'elle contient autant d'unités de traduction que de termes porteurs de sens - easy, wise, after et event, plus l'indication du temps verbal, soit cinq (correspondant à cinq nœuds dans le réseau). Mais elle en contient une autre qui recouvre l'ensemble de l'expression proverbiale (qu'on pourra éventuellement traduire par une expression proverbiale équivalente). Cette dernière unité correspond au sens rhétorique de l'expression dans le texte. Le sens rhétorique s'attache à la précaution oratoire que représente ce type de formule; elle nous permet, dans ce cas, de préciser à qui elle réfère («C'est facile pour qui de prédire le passé et qui pense cela?»). Nous avons maintenant six unités de sens 5 .

Par la méthode du découpage en unités de sens, la fidélité se mesure au nombre d'unités de sens présentes dans le texte source qu'on retrouve dans le texte cible.

C'est une méthode qui s'est avérée fiable pour l'étude de la fidélité de textes traduits entiers ${ }^{6}$ (Dancette 1990), même si elle a ses limites. En effet, elle n'est pas toujours facile à appliquer à cause du non-recoupement des notions dans les deux langues. $\mathrm{Ce}$ phénomène, lié au découpage différent de la réalité, entraine les procédés, bien connus des traducteurs, de compensation. Expliquons: on peut ne pas retrouver dans le texte cible l'expression explicite d'une unité de sens présente dans le texte source sans qu'il y ait pour autant perte ou déformation du sens, à condition qu'une autre unité soit chargée de la valeur sémantique (dénotation ou connotation) qui était attachée à la première unité. C'est ce déplacement sémantique que nous appelons compensation. Mais il s'agit là d'une limite méthodologique plutôt que théorique.

Cette approche pose néanmoins un problème fondamental : la méthode du compte des unités de sens tend à niveler les différents éléments microstructurels du texte et, par conséquent, ne permet pas de hiérarchiser les fautes.

Pour arriver à une pondération, il faut prendre un point de départ opposé, soit partir de la signification globale (macrostructurelle) du texte, au lieu de sa signification de détail (microstructurelle), car le sens d'un texte n'est pas la somme de ses éléments. La recherche sur la compréhension, dont nous exposons quelques aspects dans ce qui suit, étaye l'idée que le sens global d'un texte est ce que révèle la macrostructure de ce texte.

\section{APPRÉHENSION DU SENS}

\section{Données empiriques}

Dans une recherche expérimentale portant sur 20 répondants à qui nous avons demandé de répondre à des questions de compréhension sur un texte (test de compréhension), puis de le traduire (test de traduction), nous avons pu observer que, face à certaines difficultés de compréhension, le traducteur peut essayer de résoudre les problèmes en suivant au moins deux voies; nous les qualifions de mode centripète et de mode centrifuge de la compréhension.

Prenons la phrase (3), extraite du texte qui fait l'objet des tests:

In the post-mortem that has already started, the Democrats' right wing will press for further moderation and accommodation of the social and foreign policy prejudices of Southern white males and «Reagan Democrats». 
Nous pourrions la traduire par:

Pendant cette période des bilans qui a déjà commencé, la droite du Parti démocrate exercera des pressions dans le sens d'une politique plus modérée, d'une part, et, d'autre part, d"une plus grande adaptation aux préjugés des hommes blancs du Sud et des "démocrates reaganiens».

Cette phrase a posé des difficultés liées au découpage syntaxique de la phrase. Ce n'est cependant pas l'étude des difficultés qui retient ici notre attention, mais l'analyse des traductions. Nous en donnerons deux à titre d'exemple.

Soit la traduction (1):

Trad.(1)

Après leur cuisante défaite, l'aile droite du parti démocrate n'a pas manqué d'en analyser les causes, affirmant qu'à l'avenir, il vaudrait mieux mettre la pédale douce à certains chapitres du programme du parti, notamment en matière de réforme sociale et de politique étrangère, de façon à accommoder les hommes blancs des États du Sud et les démocrates sympathiques à Reagan.

Si on l'analyse unité sémantique par unité sémantique, en s'attachant au sens lexical des unités, à leur(s) modalité(s) et aux relations prédicatives ou actancielles liant les différentes unités dans un rapport d'argument à prédicat (voir partie II plus haut), on compte cinq erreurs :

a) sens lexical : cuisante défaite pour postmortem;

b) unité sémantique absente: that has already started;

c) sens lexical et modalités: affirmant qu'il vaudrait mieux pour will press for;

d) unité sémantique absente pour prejudices;

e) relation prédicative : mettre la pédale douce à (i)... en matière de réforme sociale et de politique étrangère (ii). L'argument (ii) ne devrait pas être attaché à (i), qui traduit moderation, mais à l'élément qui traduit prejudices;

f) relation prédicative: accommoder (i) les hommes blancs des États du Sud et les démocrates sympatiques à Reagan (ii). L'argument (ii) ne devrait pas être rattaché à $(i)$ mais à l'élément traduisant prejudices.

Par ailleurs, l'usage du verbe accommoder pour traduire to accommodate correspond à un non-sens.

Toutefois, on notera de manière intuitive que le sens global de la phrase est relativement bien traduit, malgré les erreurs relevées, à savoir l'objectif visé par la droite d'une modération de la politique du parti et l'idée de la conciliation ou du compromis avec les démocrates du Sud et les «démocrates reaganiens».

Examinons maintenant la traduction (2) d'un autre répondant. Trad.(2)

Dans le post-mortem qui a déjà commencé, l'aile droite du parti démocrate insistera pour obtenir plus de souplesse et de modération de la part des blancs du sud et des démocrates reaganistes quant à leurs préjugés sociaux et l'élaboration de leur politique étrangère.

Les erreurs sont les suivantes :

a) post-mortem pour traduire postmortem;

b) relation prédicative liant (i) de la part des blancs du Sud et des démocrates reaganistes à (ii) souplesse et modération;

c) relation prédicative liant (i) préjugés à (ii) sociaux; 
d) relation prédicative liant (i) souplesse et modération à (ii) élaboration de leur politique étrangère.

Nous notons que la traduction (2) contient un nombre relativement peu élevé de fautes et qu'à l'exception de postmortem, il n'y en a aucune de niveau lexical. Par simple calcul d'unités de sens, cette traduction devrait donc être évaluée comme supérieure à la traduction (1). Pourtant, le sens global n'y est pas du tout exprimé alors que dans la traduction (1), nous retrouvons des éléments fondamentaux du sens global. L'analyse macrostructurelle permet de rendre compte de ce phénomène.

\section{Analyse macrostructurelle des traductions}

Le sens global, à la différence du sens de détail, doit être exprimé dans la macrostructure. Comme le suggère Larose (1989), il est possible de hiérarchiser les propositions selon leur importance; les plus élevées étant celles qui charrient le plus d'information essentielle.

Ainsi, les macropropositions de la phrase (3), replacée dans son contexte minimal pour les besoins de la démonstration dans le cadre de cet article, pourraient être représentées par l'arbre qui suit ${ }^{7}$ :

\section{EXIGENCES DE LA DROITE DU PD \\ SUR LA NOUVELLE ORIENTATION DU PARTI \\ AUXQUELLES FUTURS CANDIDATS DEVRONT SE SOUMETTRE}

VIRAGE PLUS À DROITE

MODÉRATION

COMPROMIS AVEC ÉLEMENTS CONSERVATEURS

TOLÉRANCE FACE À LEURS PRÉJUGÉS

HOMMES BLANCS DU SUD

DÉMOCRATES REAGANIENS

ETC.

Si nous reprenons les traductions (1) et (2) pour comparer leur contenu macrostructurel, nous constatons qu'il n'est pas difficile de retrouver dans la première les macropropositions de niveau élevé mais qu'il est, par contre, impossible de les reconstituer à partir de la traduction (2).

\section{Approche centripète ou centrifuge du sens}

À la lumière des résultats de nos répondants aux autres tests (test de connaissance de l'anglais et test de connaissances extralinguistiques), nous pouvons dire que l'auteur de la traduction (1) est arrivé à une compréhension globale très satisfaisante du texte en dépit d'une assez faible connaissance de la langue anglaise et que l'auteur de la traduction (2) n'a pas réussi à comprendre le sens global malgré une bonne connaissance de la langue anglaise. Comment expliquer ce paradoxe apparent?

Sans entrer dans les explications sur les connaissances extralinguistiques ${ }^{8}$, nous pouvons nous hasarder à faire des hypothèses sur l'un des modes d'élaboration de la compréhension. Le traducteur (1) a procédé de manière centripète. Il est parti de sa 
connaissance du sujet, de son intuition, de sa compréhension de la politique, etc., pour en arriver à établir un sens au niveau macrostructurel sur lequel il a pu, tant bien que mal, échafauder le sens microstructurel. Le traducteur (2), qui pourtant disposait d'une meilleure connaissance du vocabulaire et apparemment d'une maîtrise satisfaisante de la syntaxe anglaise (au vu des résultats aux autres tests), a procédé de manière centrifuge, de la microstructure à la macrostructure. L'appui prioritaire sur le sens des mots du texte n'a pas suffi pour permettre la construction d'une macrostructure rendant compte du sens global du texte.

Nous illustrons ces deux démarches antagonistes au moyen des diagrammes de Venn ci-dessous.

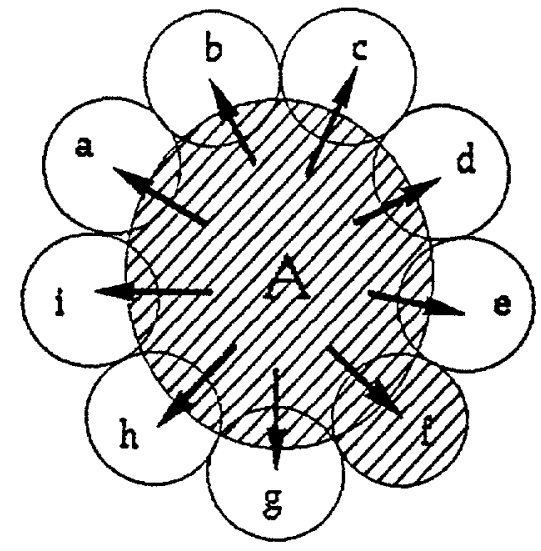

Comprehension centrifuge

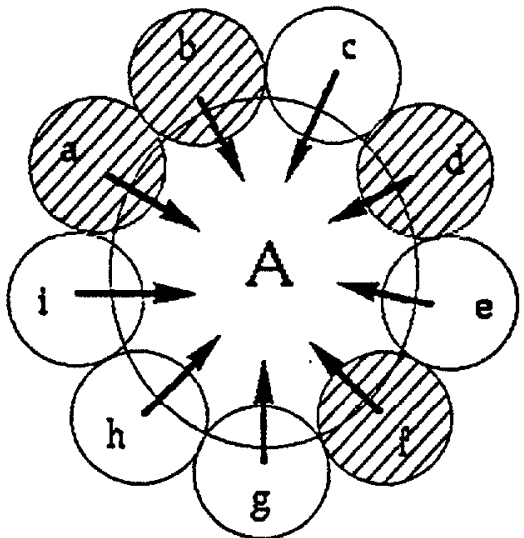

Compréhension centripète

Chaque cercle des diagrammes représente une proposition et.l'ensemble du diagramme, l'énoncé total. Le noyau est la proposition centrale qui donne le sens glolal du message, the gist of the message, ici, dans Trad.(1), l'idée de mettre la pédale douce à certains chapitres du programme du parti. Les autres cercles, qui gravitent autour du noyau central ou qui le coupent, représentent des propositions secondaires.

Théoriquement, et pour revenir au thème de cet article sur les outils de mesure de la fidélité, il est possible de quantifier la valeur relative de chaque proposition pour évaluer l'adéquation sémantique de la traduction. Ainsi, toute idée de compromis, de conciliation, de virage à droite dans les politiques des futurs candidats (contenue dans le noyau central du diagramme) pourrait avoir, par exemple, une valeur de $50 \%$, et les autres propositions $20 \%, 15 \%, 10 \%, 5 \%$, etc., selon leur importance respective. La compréhension la plus complète $(100 \%)$ sera, bien sûr, assurée par le recoupement du mouvement centrifuge et du mouvement centripète qui se renforcent l'un l'autre.

\section{CONCLUSION}

Nous avons exposé trois mesures indépendantes de la fidélité en traduction : la confrontation des relations prédicatives, l'examen des unités sémantiques et la pondération des propositions. Aucune n'est suffisante à elle seule, et même si on les utilise de façon complémentaire, elles ne rendent compte que d'un certain nombre des facteurs entrant dans la description du sens. Une autre description scientifique et complète du sens de discours en situation de communication semble impossible à l'heure actuelle et, par 
conséquent, l'analyste ne peut pas compter sur une panoplie de critères sûrs et fixes. Il se trouve un peu dans la situation du physicien en théorie quantique pour qui la vérité n'est pas absolue. S'il cherche à mesurer le mouvement d'une onde, il perd de vue son amplitude. En traduction, un phénomène analogue se produit. À trop fixer l'attention sur certains aspects du sens (l'aspect poétique ou l'expression des rapports logiques entre les propositions, par exemple), on tend à perdre un peu de vue l'aspect dénotatif du sens et vice versa. Mais le traducteur aguerri a appris à se réconcilier avec l'idée que la vérité n'est pas absolue et que l'expression du sens n'est pas parfaite. Et heureusement que sa subjectivité trouve encore sa place!

\section{NOTES}

1. Nous sommes reconnaissante à la Faculté des études supérieures de l'Université de Montréal d'avoir rendu possible cette recherche grâce à l'aide qu'elle nous a accordée.

2. Parmi d'autres critères possibles, plus ou moins pertinents selon le type des textes, tels que l'utilité pragmatique de l'information (ex.: mode d'emploi), la charge symbolique des éléments de segments d'énoncé (ex. : textes poétiques, publicitaires), etc.

3. Nous insistons sur le mot conforme; les réseaux peuvent, en effet, être conformes sans être identiques en raison de la non-isomorphie des langues en question.

4. Nous trouverions de nombreuses variantes, toutes synonymes, du genre : L'année en cours est marquée par la concurrence que se livrent les États-Unis et le Japon sur les téléviseurs couleur : les Japonais, certes, souffrent de la réévaluation du yen, mais ils ripostent en introduisant sur le marché américain des produits de grande qualité; ou bien: Les téléviseurs couleur sont l'objet cette année d'une vive concurrence. En effet, si le Japon souffre de la réévaluation du yen, il se défend énergiquement en lançant des produits de grande qualité.

5. Il n'est pas exclu qu'un traducteur traite l'expression en bloc en une seule unité de traduction, alors qu'un autre la décomposera en plusieurs unités de traduction.

6. Nous renvoyons à la présentation de la méthodologie suivie dans notre recherche de doctorat (Dancette 1990), notamment à la partie consacrée aux méthodes d'analyse des traductions au chapitre 4.

7. Nous donnons le texte complet qui faisait l'objet du test en annexe. La macrostructure n'est pas donnée telle quelle dans un texte; elle s'élabore par suite d'opérations mentales de construction de propositions et de déduction. Il s'ensuit qu'elle peut varier considérablement d'un lecteur à l'autre. Pourtant, à l'intérieur d'un groupe, si on confronte les hypothèses et qu'on en discute, un consensus peut s'établir sur le contenu macropropositionnel d'un texte ou segment de texte.

8. Voir Dancette (1990) sur l'exploitation des divers types de connaissances (linguistiques et extralinguistiques) chez les traducteurs.

\section{BIBLIOGRAPHIE}

BÉNARD, J.-P. et P. HORGUELIN (1979) : Pratique de la traduction. Version générale, Montréal, Linguatech.

BOURBEAU, L. et J. LEHRBERGER (1988): Machine Transiation: Linguistic Characteristics of MT Systems and General Methodology of Evaluation, vol. 15 de Linguistica Investigationes Supplementa, Amsterdam, John Benjamins Publishing Company.

DANCETTE, J. (1988): «Traduction-interaction : Lectures interactives et interactionnelles comme préparation à la traduction», Traduction, Terminologie et Rédaction, vol. $1, \mathrm{n}^{\circ} 2$.

DANCETTE, J. (1989): «La faute de sens en traduction, un essai de formalisation», Traduction, Terminologie et Rédaction, $\mathrm{n}^{\circ} 4$.

DANCETTE, J. (1990) : Étude réflexive et expérimentale du processus de compréhension dans l'activité de traduction, thèse de doctorat présentée à l'Université de Montréal, Département de linguistique et philologie.

DELISLE, J. (1984): L'analyse du discours comme méthode de traduction; théorie et pratique, Éditions de l'Université d'Ottawa.

GILE, D. (1987): «Modules 1 à 5», Bulletin, Japan Association of Translators, nos 22, 23, 24.

GILE, D. (1990): «La traduction et l'interprétation comme révélateurs des mécanismes de production et de compréhension du discours», Meta, 35-1.

HOUSE, J. et S. BLUM-KULKA (eds.) (1986): Interlingual and Intercultural Communication, Tübingen, Gunter Narr.

JACKENDOFF, R. (1985): Semantics and Cognition, Cambridge, The MIT Press. 
KINSTCH, W. et T. A. VAN DIJK (1975) : «Comment on se rappelle et on résume des histoires», Langages, no 40, décembre 1975, Didier, Larousse.

KINTSCH, W. et T. A. VAN DIJK (1983): Strategies of Discourse Comprehension, New York, Academic Press.

LAROSE, R. (1989): Théories contemporaines de la traduction, Presses de l'Université du Québec.

LÖRSCHER, W. (1987): Übersetzungsperformanz, Übersetzungsprozess und Übersetzungsstrategien, Habilitationsschrift, Universität Essen.

MELČUK, I. (1978) : «Théorie de langage, théorie de traduction», Meta, 23-4.

MELČUK, I. (1981): «Meaning-Text Models: A Recent Trend in Soviet Linguistics», Annual Review of Anthropology, vol. 10

NIDA, E. A. et Ch.-R. TABER (1969): The Theory and Practice of Translation, Leiden, E.J. Brill (traduction française : La traduction : théorie et méthode, Londres, Alliance biblique universelle, 1971).

POTTIER, B. (1963) : Recherches sur l'analyse sémantique en linguistique et en traduction automatique, Nancy, Université de Nancy.

SCARDAMELIA, M. et C. BEREITER (1984): «Development of Strategies in Text Processing», Mandl, Stein, Trabasso (ed.), Learning and the Comprehension of Text, Lawrence Erlbaum, pp. 379-406.

SEGUINOT, C. (1989): The Translation Process, Toronto, School of Translation, York University.

SELESKOVITCH, D. et M. LEDERER (1984): Interpréter pour traduire, Paris, Publications de la Sorbonne, Didier érudition.

WILSS, W. (1988): Kognition and Übersetzen: Zu Theorie und Praxis der menschlichen und der maschinellen Übersetzung, Tübingen, Series: Konzepte der Sprach und Literaturwissenschaft, Gen. ed. Klaus Baumgartner, Max Niemeyer.

\section{ANNEXE}

It is easy to be wise after an event. But the Jackson people can say with justification that if their man had been white, he might have pulled it off with what top adviser Bob Borosage of the Institute for Policy Studies calls his «updated version of the populist strain in Democratic Party politics» - bringing out non-voters and the excluded with an assault on the economic royalists. Jackson's reception among white, blue-collar workers shows what can be done, says Borosage.

In the post-mortem that has already started, the Democrats' right wing will press for further moderation and accommodation of the social and foreign policy prejudices of Southern white males and Reagan Democrats. A candidate for 1992 who fits that bill might be Sen. Sam Nunn or Sen. Albert Gore, who did well on Super Tuesday but proved to be no Crocodile Dundee in the jungles of New York.

- Michael White, «The Guardian», World Press Review, décembre 1988. 